\title{
Effects of radiation exposure on dermal collagen: A multi modal approach
}

Maeva, Anna, Bamber, Jeffrey, Seviaryna, Inna, Hopper, Colin, Perrett, Conal, et al.

Anna Maeva, Jeffrey Bamber, Inna Seviaryna, Colin Hopper, Conal Perrett, Laurent Bozec, "Effects of radiation exposure on dermal collagen: A multi modal approach," Proc. SPIE 11319, Medical Imaging 2020: Ultrasonic Imaging and Tomography, 113190P (16 March 2020); doi: $10.1117 / 12.2549843$

SPIE. Event: SPIE Medical Imaging, 2020, Houston, Texas, United States 


\title{
Effects of radiation exposure on dermal collagen: A multi modal approach
}

\author{
Anna Maeva*a, Jeffrey Bamber ${ }^{\mathrm{b}}$, Ina Seviaryna ${ }^{\mathrm{c}}$, Colin Hopper ${ }^{\mathrm{a}}$, Conal Perrett ${ }^{\mathrm{d}}$, , Laurent Bozec ${ }^{\mathrm{e}}$ \\ ${ }^{a}$ Eastman Dental Institute, University College London, London, UK, WC1E 6BT; ${ }^{b}$ Institute of \\ Cancer Research, Sutton, London, UK, SM2 5NG; ' Institute for Diagnostic Imaging \\ Research, University of Windsor, 688 University Ave. W., Windsor, ON Canada N9A 5R5; \\ ${ }^{\mathrm{d}}$ University College London Hospital, London, UK, NW1 2BU; ${ }^{\mathrm{e}}$ Faculty of Dentistry, University of \\ Toronto, ON, M5S, Canada
}

\begin{abstract}
Intense pulsed light (IPL) is a high-intensity treatment for skin disorders and ageing. As this treatment regime is often poorly regulated and inadequately studied, we investigate IPL as a cosmetic device and its effects on dermal collagen components of the skin.

Biopsies from the back-neck folds of a 4-week-old, $25 \mathrm{~kg}$ large white pig were irradiated with intense pulsed light (IPL) $(\lambda=584 \mathrm{~nm})$ at an increased radiation dose of $40 \mathrm{~J} / \mathrm{cm}^{2}$ once, thrice and ten times. Samples were cryo-sectioned $(10 \mu \mathrm{m})$ and stained with picro sirrus red. Ex-vivo biopsies were assessed with polarized light microscopy (PLM), atomic force microscopy (AFM) and scanning acoustic microscopy. Customized software was used to map the sound speed and attenuation on the ultrasonic images

Differences in collagen structure were observed between all three levels of irradiation progressing depth-wise into the epidermis. Ex-vivo porcine tissue demonstrated loss of D-banding and gelatinization with increasing dermal depth with higher intensities. Acoustic microscopy demonstrated a significant decrease in sound speed and attenuation that relates to the number of exposures. Sound speed decreases at much faster rates than attenuation.
\end{abstract}

Keywords: Intense pulsed light (IPL), High resolution scanning acoustic microscopy, Atomic force microscopy, Human skin, Collagen.

\section{INTRODUCTION}

Age-related changes have a deleterious impact on the function and structure of many organs of the human body, including that of the skin $^{1-3}$. From a dermatological point of view, structural changes in the skin as a function of ageing can be attributed to the slow degradation of dermal collagen associated with an increase of advanced glycation products present in the connective tissue making up the skin ${ }^{4-7}$.

Skin is a highly heterogeneous and anisotropic material which composition and structure varies with age. Collagen provides the elastic and mechanical strength to the skin. Non-invasive approaches to improve the aesthetics of skin such as laser treatments and topical creams have become very popular in the general population ${ }^{8,9}$. One rising treatment is that of Intense Pulsed Light (IPL) ${ }^{10}$. IPL is a broadband, polychromatic, high-intensity treatment capable of targeting several chromophores simultaneously. This trait makes it popular amongst practitioners since IPL can treat age spots, acne scars, remove hair and other pigmentations. Unfortunately, unlike lasers, this technique is poorly regulated and inadequately studied $^{11}$. Without proper training, severe damage and burning to dermal tissue can occur. Such types of radiative treatments are a double edge sword encompassing in them a spectrum from $400 \mathrm{~nm}$ to $1200 \mathrm{~nm}$, including UV and IR $\operatorname{light}^{12}$.

*anna.maeva.15@ucl.ac.uk;

+44 (0) 7767976714;

While light microscopy is the primary technique for evaluating structural and biochemical changes in skin biopsy specimens, assessment of mechanical properties that are relevant for the collagens condition is not possible by this method.

Medical Imaging 2020: Ultrasonic Imaging and Tomography, edited by Brett C. Byram, Nicole V. Ruiter,

Proc. of SPIE Vol. 11319, 113190P - (c) 2020 SPIE · CCC code: 1605-7422/20/\$21 · doi: 10.1117/12.2549843 
In addition to the conventional histochemical approaches, there is a need to characterize dermal structure by imaging modalities that can measure the mechanical properties of distinct tissue components. To fully understand the effect of histological changes on the mechanical properties of skin, it is essential to use techniques that assess the elastic properties of the tissue. Atomic Force Microscopy is a well-known technique for mechanical testing. Although this method offers high resolution, it is difficult to relate the observed region to traditional histology analysis. Recent advancements in Scanning Acoustic Microscopy (SAM) has induced considerable interest to this method for assessment of both histological and elastic properties using the same tissue section ${ }^{13,14}$. This technique demonstrates great concordance with histology analysis based on light microscopy ${ }^{15}, 16$. This study investigates the physical, chemical and mechanical effects of IPL and radiation on collagen fibrils in the skin.

\section{METHODOLOGY}

\subsection{Materials}

As a precursor to cross-linked ex-vivo skin samples, chemically pure, non crosslinked, Type I collagen scaffolds and solubilized single collagen molecules were irradiated with an in house IPL instrument at clinically relevant intensities and at a wavelength of $584 \mathrm{~nm}$. Solubilized liquid collagen was also prepared into a set of collagen scaffolds. Pre- and postirradiation scaffolds, as well as the solubilized collagen, were then characterized using a JPK Nanowizard 4 (Berlin, Germany) atomic force microscopy (AFM), Fourier transform infrared spectroscopy (FTIR), Philips XE FEG-SEM (Eindhoven, Netherlands) scanning electron microscopy (SEM), and high-frequency ultrasound microscopy (Honda IMS 50-SI and Tessonics) (US) to evaluate the potential structural and mechanical impact and oxidative damage incurred as a result of the irradiation.

Based on these previous results, a porcine model was chosen due to its similarity with human skin. This tissue was used to evaluate the extent of dermal collagen degradation after radiation. Biopsies from the back-neck folds of a 4-week-old, $25 \mathrm{~kg}$ large white pig were irradiated with IPL at an increased radiation dose of $48 \mathrm{~J} / \mathrm{cm}^{2}$ once, thrice and ten times. The center frequency wavelength of the IPL was that of $584 \mathrm{~nm}$. Samples were then cryo-sectioned and stained with hematoxylin and eosin or picro sirrus red at a thickness of 10 microns. The irradiated samples were characterized with AFM and optical polarized microscopy and ultrasound (US) to evaluate the potential structural and mechanical impact and oxidative damage incurred as a result of the irradiation.

\subsection{Atomic Force Microscopy}

Atomic force microscopy allows the topography and elasticity of individual collagen fibrils to be evaluated. There is quantitative imaging for AFM and qualitative indentation measurements. Each collagen fibril can be visualized with 67 $\mathrm{nm}$ banding visible. A JPK Nanowizard 4 (Berlin, Germany) was used for these measurements with MSNL-10 tips for the imaging and RSPAS for the indentation.

\subsection{Scanning Acoustic Microscopy}

Scanning acoustic microscopy (SAM) is an efficient method for evaluating mechanical properties of live tissue ${ }^{17-20}$. The technique allows non-invasive evaluation of tissue stiffness without significant tissue preparation or damage. Assuming the tissue is an isotropic material, the relation between sound speed $c$ and elastic bulk modulus $\mathrm{K}$ is:

$$
c=\sqrt{K / \rho}=\sqrt{(1+\sigma)(1-2 \sigma) E / \rho(1-\sigma)}
$$

where $\rho$-density of the tissue. E- Young's modulus, and $(\sigma)$-Poisson's ratio (approx. 0.5 in human soft tissues. The sound attenuation in the tissue relates to its viscosity:

$$
\alpha=\frac{2 f^{2} \pi^{2}}{3 \rho c^{2}}\left(\eta_{v}+\frac{4}{3} \eta_{s}\right)
$$

where $\alpha$ : absorption coefficient, $\eta_{\mathrm{v}}$ : volumetric viscosity, $\eta_{\mathrm{s}}$ : shear viscosity.

The acoustic microscope AMS-50SI (Honda Electronics) equipped with the $320 \mathrm{MHz}$ central frequency transducer was used. The analysis of the reflected wave by Fourier transform was performed to calculate the speed of sound and attenuation of sound in the tissue ${ }^{21}$. The region of interest on the acoustic images was determined using H\&E stained 
slides. The sound speed and the attenuation values were acquired from 20 pixels on each of the five chosen regions of interest.

\subsection{Optical Polarized Microscopy}

A Letiz DMRD microscope with appropriate filter and camera was used in order to view tissue samples on slides at 10micron thickness at a 10x magnification. The specimens were died with H\&E and Sirus Direct Red 80 (Sigma Aldrich, UK). Sirus staining allows for collagen to be visible under polarized light with differing degrees of degradation where green indicated healthy conditions.

\section{RESULTS}

Porcine biopsy samples were characterized from the outmost epidermal (ED) layer down to the papillary (PD) and reticular dermis (RD) to investigate the extent of radiation damage to collagen fibrils. The ED layers were directly exposed to IPL followed closely by PD. Differences in the structural morphology and orientation of the collagen were observed for all of the irradiated porcine skin model tissue when compared with the controls of each layer of non-irradiated skin.

Polarized light microscopy showed distinct signs of collagen damage with increasing radiation dose where porcine skin exposed to $48 \mathrm{~J} / \mathrm{cm} 2$ was observed to be a vivid purple colour compared to the standard green and yellow in the control, symbolizing a disturbance in the collagen fibril orientation.

Acoustic microscopy presents additional information complementary to light microscopy. The contrast between the papillary and reticular dermis often is notably distinct. A clear difference in skin structure and its properties are evident as a result of increasing IPL irradiation (Figure 1). The transformation in collagen structure due to the increased irradiation time is evident in the intensity images (Figure 1A and Figure 2). Sound speed and attenuation mapping also supports the alterations in the collagen biomechanics. The gradual change in mapping colours from red-yellow to green-blue indicates a significant decline in sound speed and attenuation values. Table 1 shows changes in acoustic properties of the porcine skin after IPL irradiation. Both sound speed and attenuation decreases with higher exposure. The sound speed decreases gradually in skin samples irradiated one, three and ten times. The attenuation demonstrates more powerful decline in value compared with the sound speed. This parameter gradually decreases after the one irradiation and falls drastically after ten exposures. The biological meaning of changes in the acoustic properties needs further studies.

The AFM analysis of the irradiated porcine skin (Figure 3) confirms the significant changes in the collagen structure caused by the IPL. The collagen is significantly degraded and gelatinized. The high-resolution ultrasonic images of the comparable skin region also indicate both changes in collagen structure and diminution of sound speed and attenuation values.

Overall damage to samples that were only radiated once was only visible at the most epidermal layer, whereas tissue irradiated thrice demonstrated areas of damage to the PD and beginning to the RD as well. The porcine tissue irradiated ten times resembled what would be found in parchment with signs of gelatinization and loss of banding, essential to collagen fibril and skin structure. The loss of these crucial markers of collagen health suggests clear and extensive damage to the skin after irradiation, as seen in AFM images Figure 1, which also correlated with US scans. Mechanical testing demonstrated an increase in Young's (elastic) modulus with increased irradiation due to pathological cross-linking effects and dehydration. Ultrasound data showed an increase in the density of the tissue during irradiation. 
1

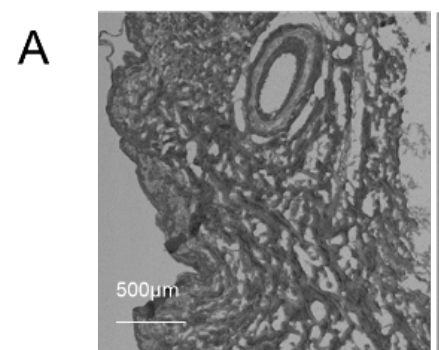

B

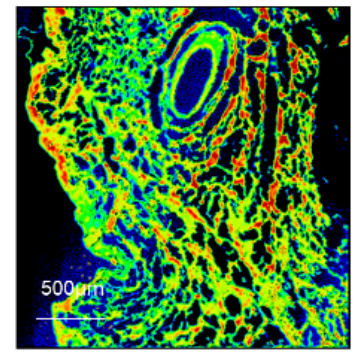

C

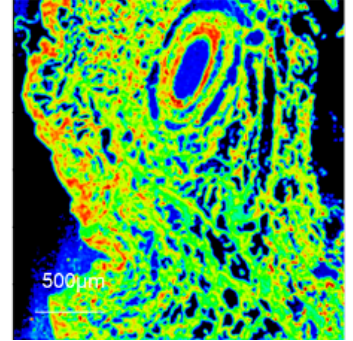

2
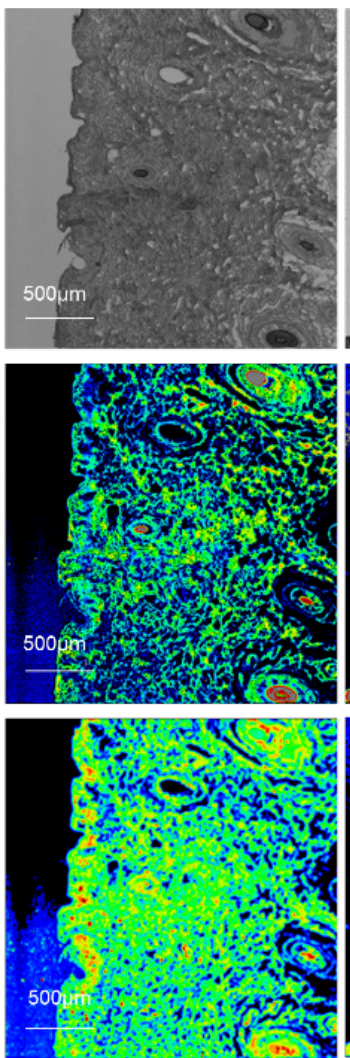

3
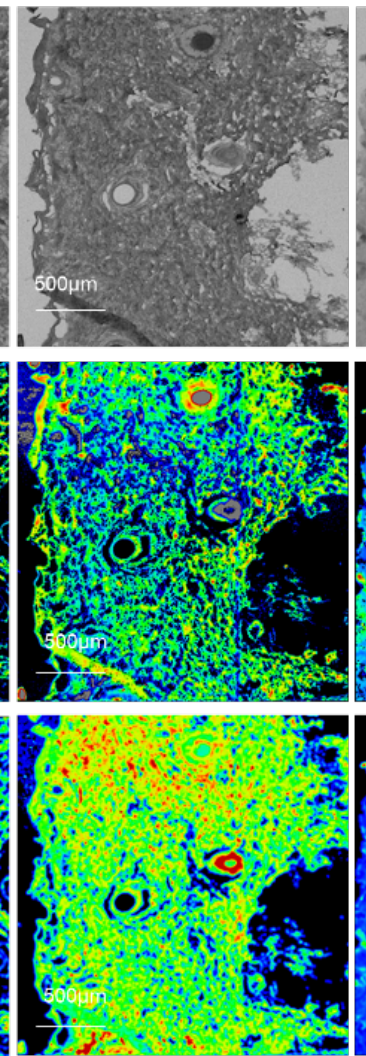

4
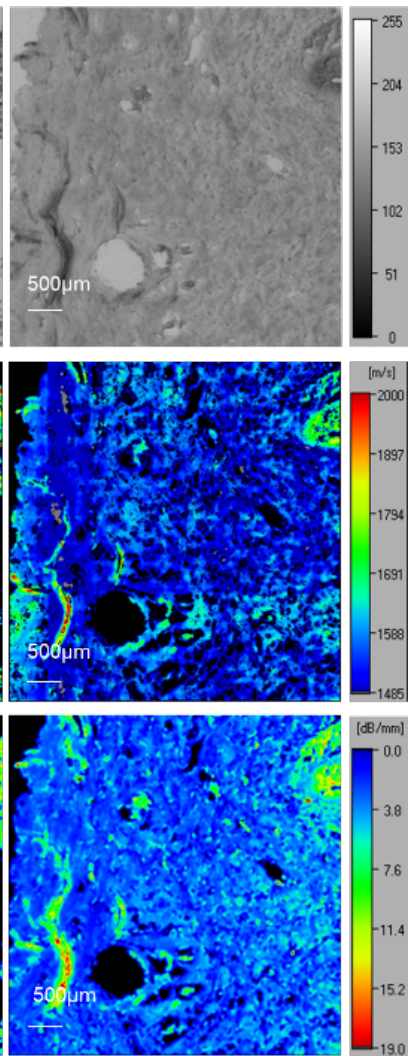

Figure 1. SAM images of $40 \mathrm{~J} / \mathrm{cm}^{2}$ IPL irradiation porcine skin. (A) acoustic intensity, (B) Sound speed, (C) attenuation mapping. The skin samples was exposed: 1 - no exposure $(0), 2$ - one time $(1 \mathrm{x}), 3$ - three times $(3 \mathrm{x}), 4-\operatorname{ten}$ times $(10 \mathrm{x})$.
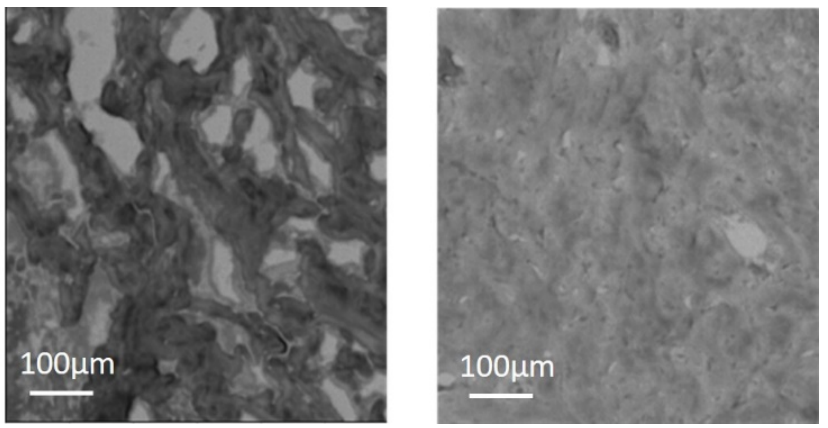

Figure 2. SAM images of porcine skin's papillary dermis. (a) non-irradiated, (b) irradiated with IPL $40 \mathrm{~J} / \mathrm{cm} 2 \mathrm{x} 10 \mathrm{dose}$.

Thus, both polarized light, AFM imaging and qualitative SAM images indicated that collagen fibers degrade depth-wise into the reticular dermis. At maximum irradiation, loss of banding and gelatinisation was observed at AFM and SAM images. Damaged collagen demonstrated much lower sound speed and attenuation compared to non-irradiated skin. Apparent decreasing differences in measurements of sound speed and attenuation are evident. Although collagen degradation plays an essential role in ageing, the mechanism of this phenomenon is not fully understood yet. Studies report the several effects on the collagen structure: depolymerization, enhanced or reduced crosslinking, fiber re-orientation ${ }^{22,23,}$ collagen denaturation and dermal matrix amorphization, splitting of collagen bundles ${ }^{24}$. 
Table 1. Change in acoustic properties of the porcine skin after IPL irradiation $(40 \mathrm{~J} / \mathrm{cm} 3)$.

\begin{tabular}{|c|c|c|c|c|}
\hline $\begin{array}{c}\text { Multiplicity of IPL } \\
\text { irradiation }\end{array}$ & $\mathbf{0}$ & $\mathbf{1}$ & $\mathbf{3}$ & $\mathbf{1 0}$ \\
\hline \multicolumn{6}{|c|}{ Sound Speed, \% } \\
\hline $\begin{array}{c}\text { Papillary Dermis } \\
\text { Deticular }\end{array}$ & 100 & 92 & 87 & 85 \\
\hline \multicolumn{7}{|c|}{ Sound Attenuation, \% } & 87 & 18 \\
\hline $\begin{array}{c}\text { Papillary Dermis } \\
\text { Reticular }\end{array}$ & 100 & 93 & 82 & 44 \\
\hline
\end{tabular}

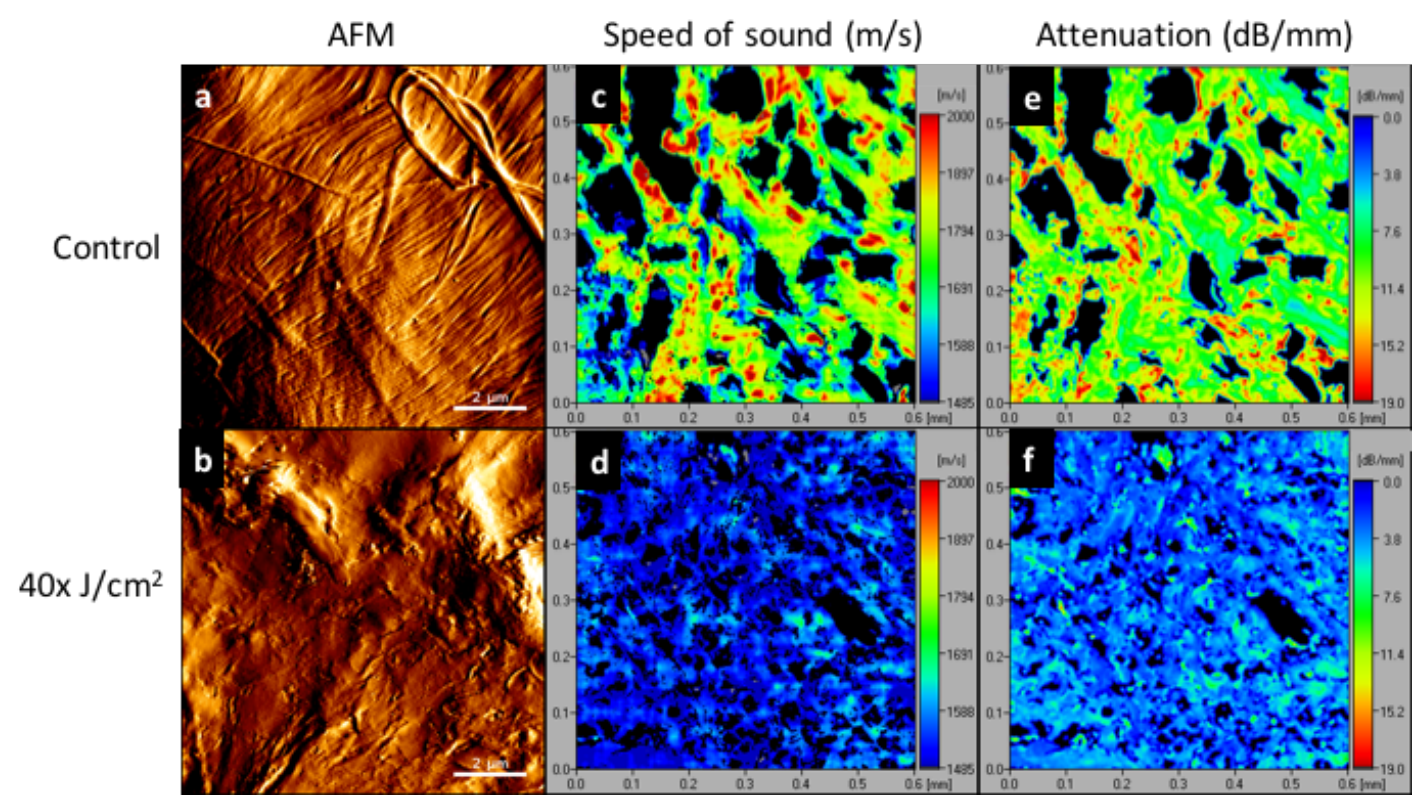

Figure 3. (a) AFM image of control porcine skin. (b) AFM image of RD of 10x $40 \mathrm{~J} / \mathrm{cm}^{2}$ irradiated porcine skin. (c) Acoustic microscopy speed of sound the intact porcine skin (e) Acoustic microscopy attenuation on the control and (d) porcine skin irradiated $10 \mathrm{x}$ of $40 \mathrm{~J} / \mathrm{cm}^{2}$ sound speed and (f) porcine skin irradiated 10x of $40 \mathrm{~J} / \mathrm{cm}^{2}$ sound attenuation.

\section{CONCLUSIONS}

The effect of intense pulsed light (IPL) on porcine skin was investigated on the molecular level using the multimodal approach. Besides imaging, mechanical properties of skin were assessed as a function of the IPL exposure. This study has permitted the investigation of radiation-induced oxidative damage to one of the most abundant and crucial proteins in the human body. Considering that this damage occurs as a result of IPL exposure, which has little regulation, it is paramount that its effects are well documented and strict guidelines are laid out for its cosmetic and medical uses. This study also provides insight into the accelerated light damage occurring to the skin and its dermal sub-layers giving valuable insight into ageing and disease formation and inflammation as a study of the mechanical and structural properties of the skin. 


\section{REFERENCES}

[1] Takema, Y., Yorimoto, Y., Kawai, M., Imokawa, G., "Age-related changes in the elastic properties and thickness of human facial skin," British Journal of Dermatology 131 (5), 641-648 (1994).

[2] Krueger, N., Luebberding, S., Oltmer, M., Streker, M., Kerscher, M., "Age-related changes in skin mechanical properties: a quantitative evaluation of 120 female subjects," Skin Research and Technology 17 (2), 141-148 (2011).

[3] Luebberding, S., Krueger, N., Kerscher, M., "Age-related changes in skin barrier function-Quantitative evaluation of 150 female subjects," International Journal of Cosmetic Science 35, 183-190 (2013).

[4] Bailey, A. J., Shimolomaki, M. S., "Age-related changes in the reducible cross-links of collagen," FEBS Letters 16 (2), 86-88 (1971).

[5] Haydont, V., Bernard, B., Fortunel, N., "Age-related evolutions of the dermis: Clinical signs, fibroblast and extracellular matrix dynamics," Mechanisms of Ageing and Development 177 (1), 150-156 (2019).

[6] Yasui, T., Yonetsu, M., Tanaka, R., Tanaka, Y., Fukushima, S., Yamashita, T., Ogura, Y., Hirao, T., Murota, H., Araki, T., "In vivo observation of age-related structural changes of dermal collagen in human facial skin using collagen-sensitive second harmonic generation microscope equipped with 1250-nm mode-locked Cr:Forsterite laser," Journal of Biomedical Optics 18 (3), 031108 (5 December 2012).

[7] Wu, S., Li, H., Yang, H., Zhang, X., Li, Z., Xu, Z., "Quantitative analysis on collagen morphology in aging skin based on multiphoton microscopy," Journal of Biomedical Optics 16 (4), 040502 (2011).

[8] Mazzoni, D., Lin, M. J., Dubin, D. P., Khorasani, H. "Review of non-invasive body contouring devices for fat reduction, skin tightening and muscle definition," Australasian journal of Dermatology https://doi.org/10.1111/ajd.13090, (2019).

[9] Ghani, S. "Review of non-invasive and minimally invasive skin tightening techniques," Journal of Aesthetic Nursing 8 (Sup1) (2019).

[10] Yagerman, S. E. and Goldberg, D. J. "Facial Skin Rejuvenation with IPL" in Fodor L., Ullmann Y. (eds) [Aesthetic Applications of Intense Pulsed Light], Springer, Cham, 49-58 (2020).

[11] Chen, M.-F., Tsai, C.-L., Chen, Y.-H., u, C.-N., Chou, C., Chien, C.-H., Tu, P.-W., Kao, T., Lin, K.-P. “Comparative Study of Regulations of Devices for Aesthetic Purposes: US, European Union, China, and Taiwan," Journal of Medical and Biological Engineering, https://doi.org/10.1007/s40846-019-00486-7, (2019).

[12] Fodor, L., Bota, O. "Complications After IPL Treatment" in: Fodor L., Ullmann Y. (eds) [Aesthetic Applications of Intense Pulsed Light], Springer, Cham, 195-216 (2020).

[13] Miura, K., Yamashita, K., "Evaluation of aging, diabetes mellitus, and skin wounds by scanning acoustic microscopy with protease digestion," Pathobiological Aging Age Related Diseases 8(1), 1-12 (2018).

[14] Bilen, B., Sener, L. T., Albeniz, I., Sezen, M., Unlu, M. B., Ugurlucan, M., "Determination of Ultrastructural Properties of Human Carotid Atherosclerotic Plaques by Scanning Acoustic Microscopy, Micro-Computer Tomography, Scanning Electron Microscopy and Energy Dispersive X-Ray Spectroscopy,” Scientific Reports, 9, 679 (2018).

[15] Youssef, S., Seviaryna, I., Shum, D., Maeva, E., Malyarenko, E., Rahman, N., Maev, R. G., "High-resolution quantitative acoustic microscopy of cutaneous carcinoma and melanoma: Comparison with histology," Skin Research and Technology 25(5), 662-671 (2019).

[16] Winterroth, F., Hollman, K. W., Kuo, S., Izumi, K., Feinberg, S. E., Hollister, S.J., Fowlkes, J. B., “Comparison of scanning acoustic microscopy and histology images in characterizing surface irregularities among engineered human oral mucosal tissues," Ultrasound IN Medicine and Biology 37 (10), 1734-1742 (2011).

[17] Maev, R. G., "Investigation of the Microstructure and Physical-Mechanical Properties of Biological Tissues," In [Acoustic Microscopy: Fundamentals and Applications], Wiley-VCH, Weinheim, Germany, 187-241 (2008).

[18] Saijo, Y., Tanaka, M., Okawai, H., Sasaki, H., Nitta, S. I., and Dunn, F. "Ultrasonic tissue characterization of photodamaged skin by scanning aocutsic microscopy,” Tokai J Exp Clin Med. 30 (4), 217-225 (2005).

[19] Cantrell Jr, J. H., Goans, R. E. and Roswell, R. L. "Acoustic impedance variations at burn-nonburn interfaces in porcine skin," J. Acoust. Soc. Am. 64, 731-738 (1978).

[20] Saijo, Y. "Three-dimensional Ultrasound Imaging of Regenerated Skin with High Frequency Ultrasound," Proc. $5^{\text {th }}$ IEEE International Symposium on Biomedical Imaging: From Nano to Macro, 1231-1234 (2008).

[21] Hozumi N, Yamashita R, Lee CK, et al. Time-frequency analysis for pulse driven ultrasonic microscopy for biological tissue characterization. Ultrasonics 42(1-9), 717-722 (2004). 
[22] Manickavasagam, A, Hirvonen, L. M., Melita, L. N., Chong, E. Z., Cook, R.J., Bozec, L, Festy, F. "Multimodal optical characterisation of collagen photodegradation by femtosecond infrared laser ablation," Analyst 139 (23), 61356143 (2014).

[23] Ignatieva, N., Zakharkina, O., Dadasheva, A. Shekhter, A., Sviridov, A., Lunin, V., "Transformation of the dermal collagen framework under laser heating," Journal of Biophotonics, https://doi.org/10.1002/jbio.201960024 (2019).

[24] Markel, M. D., Hayashi, K., Thabit, G. "Basic Properties of Collagen Shrinkage and Laser-Collagen Interactions in Gerber B.E., Knight M.T.N., Siebert W.E. (eds) Lasers in the Musculoskeletal System. Springer, Berlin, Heidelberg, 162-169 (2001). 